\title{
Long-Term Plasticity of the Spinal Locomotor Circuitry Mediated by Endocannabinoid and Nitric Oxide Signaling
}

\author{
Alexandros Kyriakatos and Abdeljabbar El Manira \\ Nobel Institute for Neurophysiology, Department of Neuroscience, Karolinska Institutet, SE-171 77 Stockholm, Sweden
}

Retrograde signaling by endocannabinoids is known to induce short- and long-term synaptic plasticity, but the significance of this modulation for the activity of neural networks underlying motor behavior is largely unclear. Here, we used the isolated lamprey spinal cord to show that endocannabinoids released by activation of metabotropic glutamate receptor 1 (mGluR1) induce long-term synaptic plasticity during an ongoing locomotor rhythm and how this is translated onto the integrated activity of the spinal circuitry. A brief activation of mGluR1 induces a long-term increase in the locomotor frequency that is mediated by a concomitant long-term depression of midcycle reciprocal inhibition and long-term potentiation of ipsilateral synaptic excitation arising from locomotor circuit interneurons. Blockade of cannabinoid receptors with AM251 prevented the mGluR1-mediated long-term plasticity of both inhibitory and excitatory synaptic transmission, as well as that of the locomotor activity. Similarly, inhibition of nitric oxide signaling blocked the mGluR1-mediated long-term plasticity. These results show that the locomotor circuitry is endowed with a "memory" capacity mediated by a long-term shift in the balance between synaptic inhibition and excitation. This is triggered by activation of mGluR1 and requires subsequent endocannabinoid and nitric oxide signaling.

Key words: locomotion; spinal cord; modulation; mGluRs; lamprey; endocannabinoids; plasticity

\section{Introduction}

Activity-dependent plasticity of synaptic transmission is believed to play a critical role adapting nervous system activity to changes in the internal and external environment (Abbott and Regehr, 2004; Destexhe and Marder, 2004; Dan and Poo, 2006). Diverse mechanisms have been shown to underlie short- and long-term changes in the strength of synaptic transmission (Malenka and Bear, 2004), including retrograde signaling by endocannabinoids (Wilson and Nicoll, 2002; Chevaleyre et al., 2006; Safo et al., 2006) and nitric oxide (Micheva et al., 2003), in various regions of the CNS (Garthwaite and Boulton, 1995; Prast and Philippu, 2001; Freund et al., 2003; Chevaleyre et al., 2006). Studies using slice preparations have provided insights into the mechanisms used by endocannabinoids to depress synaptic transmission (Kreitzer and Regehr, 2001; Wilson et al., 2001; Freund et al., 2003; Chevaleyre et al., 2006; Safo et al., 2006). However, little is known about how the endocannabinoid- and nitric oxidemediated synaptic plasticity is translated onto the activity of neural circuits underlying motor behavior. This is because most of these studies have used quiescent slice preparations where the behavioral relevance of the neural circuitry activity is difficult to establish.

\footnotetext{
Received July 12, 2007; revised Sept. 18, 2007; accepted Sept. 26, 2007.

This work was supported by the Swedish Research Council, Christopher and Dana Reeve Foundation and Karolinska Institutet. We thank Drs. Sten Grillner, Jens Peter Gabriel, and Russell Hill for their comments on this manuscript.

Correspondence should be addressed to Abdeljabbar El Manira, Nobel Institute for Neurophysiology, Department

of Neuroscience, Karolinska Institutet, SE-171 77 Stockholm, Sweden. E-mail: Abdel.EIManira@ki.se. D0I:10.1523/JNEUROSCI.3174-07.2007

Copyright $\odot 2007$ Society for Neuroscience $\quad$ 0270-6474/07/2712664-11\$15.00/0
}

Neural networks underlying rhythmic motor behaviors, such as locomotion or respiration, are more amenable to linking changes in cellular and synaptic activity to the integrated function of the networks (Feldman et al., 2003; Grillner, 2003; Ramirez and Viemari, 2005; Kiehn, 2006; Marder and Goaillard, 2006). These networks produce rhythmic motor patterns corresponding to a well defined behavior that can be readily monitored together with the synaptic activity of the constituent neurons. The locomotor networks are located in the spinal cord and are subject to extrinsic and intrinsic modulation (McLean et al., 2000; El Manira et al., 2002; Grillner, 2003; Kiehn, 2006). In this study, we have examined the role of endocannabinoids and nitric oxide in mediating long-term plasticity of the locomotor activity and have analyzed the synaptic mechanisms responsible. We have used the lamprey spinal cord in which endocannabinoids, released by activation of metabotropic glutamate receptor 1 (mGluR1), act as an intrinsic modulator (Kettunen et al., 2005). In the lamprey (Buchanan, 1982; Buchanan and Grillner, 1987; Grillner, 2003), as in Xenopus tadpoles (Dale, 1985; Dale and Roberts, 1985), the basic organization of the spinal locomotor circuit has been characterized. The fact that excitatory and inhibitory interneurons are activated in different phases of the locomotor cycle allows for monitoring their synaptic transmission during ongoing network activity. The combined analysis of modulation of synaptic transmission from identified classes of interneurons and the locomotor activity in which they participate, will allow synaptic plasticity to be linked to changes in the operation of the spinal networks.

The present study shows that a brief activation of mGluR1 induces long-term potentiation of the locomotor circuit activity. This modulation is mediated by a long-term potentiation of ipsilateral excitation in parallel with a long-term depression of mid- 
cycle inhibition, both of which require release of not only endocannabinoids, but also of nitric oxide. Thus, activation of mGluR1 recruits both endocannabinoids and nitric oxide signaling to shift the balance between inhibition and excitation in the locomotor circuitry and thereby induce the long-term increase in the activity of the locomotor network.

\section{Materials and Methods}

Experiments were performed in vitro using the intact spinal cord from adult lampreys (Lampetra fluviatilis). All protocols were approved by the animal research ethical committee, Stockholm. Lampreys were anesthetized with MS 222 (100 mg/l; Sigma, St Louis, MO), eviscerated and the lateral muscle walls were removed. The spinal cord and notochord were dissected and pinned in a cooled $\left(8-12^{\circ} \mathrm{C}\right)$ Sylgard-lined experimental chamber continuously perfused with physiological solution. The control solution was composed of (in mM) $138 \mathrm{NaCl}, 2.1 \mathrm{KCl}, 1.8 \mathrm{CaCl}_{2}, 1.2$ $\mathrm{MgCl}_{2}, 4$ glucose, 2 HEPES, bubbled with $\mathrm{O}_{2}$ and $\mathrm{pH}$ adjusted to 7.4. To analyze the changes in the locomotor frequency, the spinal cord was left attached to the notochord and the meninges were not removed. In this way, the ventral roots were left intact for long-lasting recording $(>24 \mathrm{~h})$ without any damage in the ventral roots. Fictive swimming activity was induced by adding 50-100 $\mu \mathrm{M}$ NMDA (Tocris, Bristol, UK) (Grillner et al., 1981) to the physiological solution. Alternating locomotor burst activity was recorded by en-passant glass suction electrodes placed on two opposing ventral roots at their exits from the spinal cord. After perfusion of NMDA, the locomotor burst frequency increased gradually over time and reached a stable level after 3-4 h. Agonists and antagonists were only added after the burst frequency had been stable for at least $1 \mathrm{~h}$. In preincubation experiments, the spinal cord was perfused with cannabinoid receptor, mGluR1, or nitric oxide blockers for $1-2 \mathrm{~h}$ before application of NMDA. Two minute recordings of ventral root activity were sampled every $5 \mathrm{~min}$. The cycle duration was measured and averaged from $100-$ 200 consecutive cycles. The cycle duration was defined as the time interval between the onsets of two consecutive bursts and the burst frequency was calculated as the inverse of the averaged cycle duration.

A brainstem-spinal cord preparation was used to study the effect of synaptically released glutamate on the locomotor frequency. In these experiments, the recording chamber was separated into two pools. The brainstem and the rostral spinal cord were perfused with a $\mathrm{Ca}^{2+}$-free solution containing AP5 and CNQX to block synaptic transmission in the rostral pool. The caudal spinal cord was perfused with NMDA and locomotor activity was monitored by recording two opposing ventral roots in the caudal pool. A suction electrode was placed at the junction between the brainstem and the spinal cord to stimulate descending reticulospinal axons.

To examine the changes in midcycle reciprocal inhibition during locomotion, a notochord/spinal cord preparation was used in which the dorsal meninges were removed. Intracellular recordings were made from motoneurons (MNs), commissural interneurons (crossing caudally projecting, CC-IN), and ipsilateral network interneurons with $3 \mathrm{M}$ potassium acetate-filled thin-walled glass microelectrodes with a resistance of 15-30 $\mathrm{M} \Omega$. Motoneurons were identified by recording their axonal action potentials in a one-to-one manner from the corresponding ventral root through the extracellular suction electrode. Commissural network interneurons were identified by recording extracellularly their axonal action potentials 2-3 segments caudal and contralateral to the intracellular recording site. All neurons recorded during fictive locomotion received phasic excitation alternating with phasic inhibition. The concurrent recordings of the locomotor frequency and of the activity of motoneurons and network interneurons allowed us to correlate changes in the synaptic amplitude to those in locomotor frequency. Two minute recordings of intracellular and extracellular activity recordings were sampled every 5 min. The spikes were removed using a low-pass filter and the synaptic input was averaged from 100-200 cycles and their duration was normalized. The peak to trough amplitude of the locomotor-mediated synaptic input was measured and monitored in control, in $(R S) 3,5$ dihydroxyphenylglycine (DHPG), and after washout.

To examine the effects of DHPG on ipsilateral excitatory or crossing inhibitory synaptic transmission the recording chamber was divided into two pools by an agar barrier (Dale and Grillner, 1986). Locomotor activity was induced in the rostral pool by NMDA $(100 \mu \mathrm{M})$ while the spinal cord in the caudal pool was perfused with physiological solution containing either strychnine $(5 \mu \mathrm{M})$ to block inhibitory synaptic transmission or kynurenic acid ( $2 \mathrm{~mm}$ ) to block ionotropic glutamate receptors. After 30 min in strychnine, intracellular recordings were made from motoneurons, 2-3 segments away from the agar barrier. In the presence of strychnine, excitatory synaptic input occurred in phase with the activity of the ipsilateral ventral root in the rostral pool. Inhibitory synaptic input was recorded in the presence of kynurenic acid and occurred in phase with the contralateral ventral root. Two minute recordings of intracellular and extracellular activities were sampled every $4 \mathrm{~min}$ and the amplitude the excitatory or inhibitory input was monitored in control, in DHPG, and after washout.

The effect of DHPG on excitatory synaptic transmission was also examined in quiescent preparations. The spinal cord was completely isolated and a stimulating suction electrode was placed in the ventral surface, ipsilateral to the recording site, to activate axons of excitatory interneurons and reticulospinal neurons. Intracellular recordings were made from identified MNs and monosynaptic EPSPs were recorded in a high- $\mathrm{Ca}^{2+} /$ high $\mathrm{Mg}^{2+}$ solution to minimize polysynaptic transmission. Both strychnine and AP5 were added to this solution to block glycine and NMDA receptors, respectively.

Intracellular recordings were made in bridge mode current-clamp mode with an Axoclamp 2B amplifier (Molecular Devices, Foster City, $\mathrm{CA}$ ). Molecular Devices software (pClamp) was used for data acquisition and analysis on a personal computer equipped with an analog-digital interface (Digidata 1300). The values reported correspond to mean \pm SEM and $n$ represents the number of experiments. The statistical significance of the difference between control and preparations preincubated with different antagonists was assessed using one-way ANOVA with repeated measures and $p$ values were obtained by post hoc analyses using the Dunnett's test for multiple comparisons between the different treatments. In some cases the significance was assessed with paired or unpaired Student's $t$ test. Differences were considered significant if $p<$ 0.05. All data analysis was done off line with the program Spike 2 (CED, Cambridge, UK).

The following drugs were used in this study: NMDA (100 $\mu \mathrm{M}$; Tocris), strychnine ( $5 \mu \mathrm{M}$; Sigma, St. Louis, MO), the group I mGluR agonist DHPG (100 $\mu \mathrm{M}$; Tocris), the mGluR1 antagonist 7-(hydroxyimino)cyclopropa [b]chromen-1a-carboxylate ethyl ester (CPCCOEt; $100 \mu \mathrm{M}$; Tocris), the cannabinoid receptor antagonist AM251 (1-2 $\mu \mathrm{M}$; Tocris), the nonselective nitric oxide synthase inhibitor NG-nitro-L-arginine methyl ester (L-NAME; $100 \mu \mathrm{M}$; Tocris), the nitric oxide scavenger 2-(4-carboxyphenyl)-4,4,5,5tetramethylimidazoline-1-oxyl-3-oxide (PTIO; $200 \mu \mathrm{M}$; Tocris), the inhibitor of nitric oxide-sensitive guanylyl cyclase $1 H$-[1,2,4] oxadiazolo[4,3,-a] quinoxalin-1-one (ODQ; $5 \mu \mathrm{M}$; Tocris), the NMDA receptor antagonist $\mathrm{D}(-)$-2-amino-5-phosphonopentanoic acid (AP5; $50 \mu \mathrm{M}$; Tocris), 6-cyano7-nitroquinoxaline-2,3-dione (CNQX; $20 \mu \mathrm{M}$; Tocris), and kynurenic acid (KYAC; 2 mM; Sigma). All agonists and antagonists were dissolved as stock solutions in water except CPCCOEt and AM-251 that were dissolved in DMSO. The same concentration of the solvent DMSO had no effect on the locomotor frequency and was always added to control solutions throughout the experiments.

\section{Results}

mGluR1 induces a short- and a long-term potentiation of the locomotor frequency

To examine if activation of group 1 mGluRs induces long-term potentiation of the locomotor frequency, the agonist DHPG (100 $\mu \mathrm{M}$ ) was applied for $20 \mathrm{~min}$ and its effect was monitored continuously over several hours. The locomotor rhythm was induced by application of NMDA $(100 \mu \mathrm{M})$ (Fig. $1 A)$ and DHPG was added only after the baseline locomotor frequency had been stable for at least $1 \mathrm{~h}$. Application of DHPG induced a marked increase in the locomotor frequency that displayed two time courses, a short- 
A vr-left
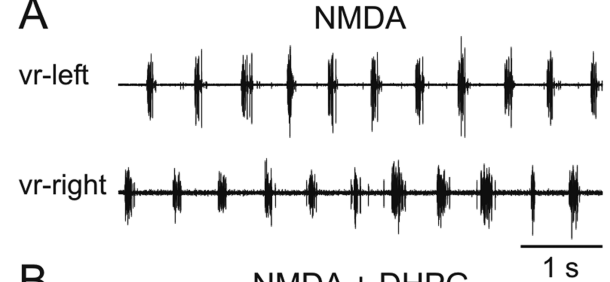

B
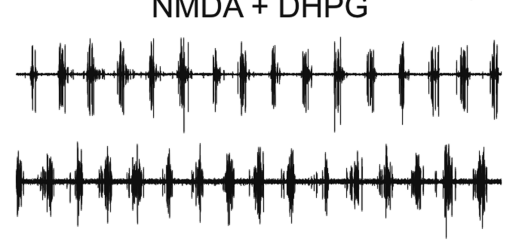

C
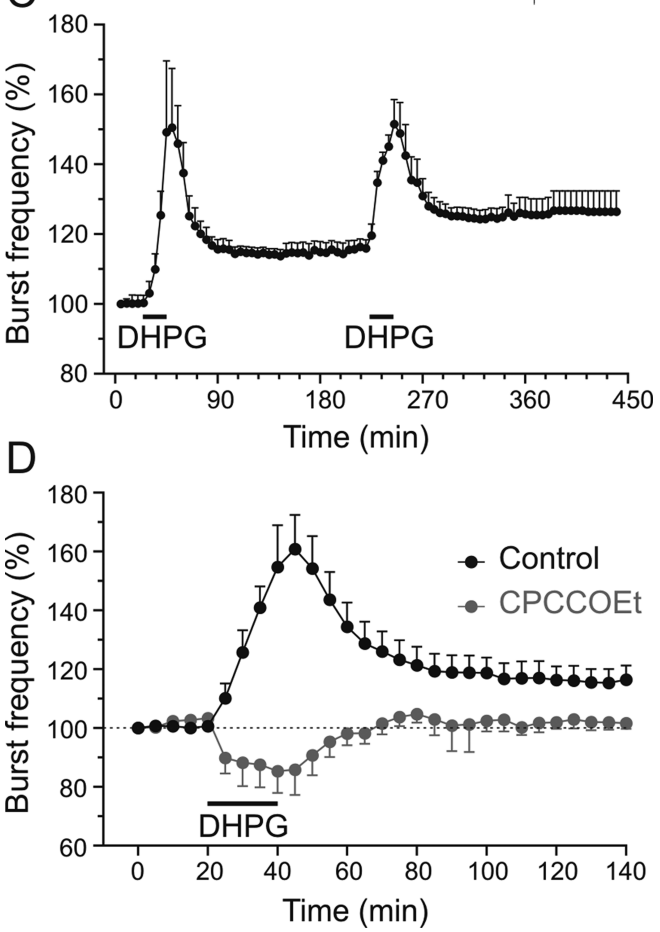

Figure 1. Short- and long-term potentiation of the locomotor circuit activity. $A$, Alternating locomotor burst induced by NMDA $(100 \mu \mathrm{M})$ recorded from two opposing ventral roots in the same segment (vr-left and vr-right). $\boldsymbol{B}$, Activation of mGluR1 by DHPG (100 $\mu \mathrm{M})$ increased the locomotor burst frequency. $C$, Plot of averaged data from different preparations showing the time course of the increase in the locomotor burst frequency induced by two successive applications of DHPG for 20 min (indicated with the bar). The first application of DHPG induced a short- and long-term increase in the frequency. The second application of DHPG induced a similar short-term effect and further enhanced the long-term increase in the frequency compared with the first application. D, Graph showing the effect of DHPG on the locomotor burst frequency in control and in preparations preincubated with the mGluR1 antagonist CPCCOEt $(100 \mu \mathrm{m})$.

and a long-term potentiation (Fig. $1 B, C$ ). The short-term potentiation of the frequency was seen during the perfusion of DHPG and amounted to $150.6 \pm 16.9 \%$ of $\operatorname{control}(n=6 ; p<0.01)$ (Fig. $1 C)$, confirming our previous results (Krieger et al., 1998, 2000). The long-term potentiation was $116.3 \pm 2.2 \%$ of control $(n=6$; $p<0.01$ ) (Fig. 1C) and lasted for $>24 \mathrm{~h}$ after washout of DHPG (data not shown). A second application of DHPG after $3 \mathrm{~h}$ washout of the first application induced short-term potentiation of the locomotor frequency by $151.5 \pm 7.0 \%$ of control $(n=6 ; p<$ $0.01)$ which was not significantly $(p>0.05)$ different from that of the first DHPG application (Fig. 1C). The long-term potentiation induced by the second application of DHPG was $126.5 \pm$
$5.9 \%$ of control $(n=6)$ and reached a significantly $(p<0.05)$ higher plateau than that induced by the first application DHPG (Fig. 1C). Both the short- and long-term potentiation induced by DHPG was blocked by mGluR1 subtype specific antagonist CPCCOEt $(100 \mu \mathrm{M})(n=5)$ (Fig. $1 D)$ (Krieger et al., 1998, 2000). In the presence of CPCCOEt, DHPG only induced a transient decrease in the locomotor frequency that is likely to be mediated by activation of mGluR5 which was shown previously to depress the frequency (Kettunen et al., 2002). These results show that DHPG acts on mGluR1 to induce not only a transient increase in the locomotor frequency, but it also mediates long-term potentiation of the activity of the locomotor circuitry.

Endocannabinoids released by activation of mGluR1 mediate the long-term potentiation

We showed previously that activation of group 1 mGluRs induces release of endocannabinoids from locomotor network neurons (Kettunen et al., 2005). To test whether the long-term potentiation of the locomotor frequency induced by activation of mGluR1 also involves release of endocannabinoids within the locomotor circuitry, the effect of DHPG was tested in preparations preincubated with the cannabinoid receptor antagonist AM-251 $(1 \mu \mathrm{M})$. In control preparations, a 20 min application of DHPG always induced a short- and long-term potentiation of the locomotor frequency (Fig. $2 A$ ). The short-term increase in the frequency was $156.7 \pm 14.2 \%$ of control $(n=8)$, whereas the long-term effect was $118.2 \pm 3.5 \%$ of control $(n=8)$ and persisted for several hours after washout of DHPG (Fig. 2C). In preparations preincubated with AM-251 (1 $\mu \mathrm{M}, 2 \mathrm{~h})$, application of DHPG induced only the short-term increase in the locomotor frequency ( $128.6 \pm 4.2 \%$ of control; $n=8)$ while the long-term effect was completely blocked (Fig. 2B). The DHPG-induced short-term increase in the frequency was significantly smaller in AM-251 than in control preparations $(p<0.01)$. The frequency always recovered after washout of DHPG $(94.9 \pm 2.4 \%$ of control; $n=8$ ) (Fig. 2C). These results confirm that a component of the short-term modulation is also sensitive to AM-251 (Kettunen et al., 2005). In addition, they show that the long-term potentiation of the locomotor frequency by mGluR1 is dependent on release of endocannabinoids within the spinal circuitry.

Glutamate release from excitatory axons induces long-term potentiation of locomotor activity

To test whether activation of mGluR1 by glutamate release from excitatory axons is able to mediate long-term potentiation of the locomotor frequency, a brainstem-spinal cord preparation was used. This preparation was mounted in a recoding chamber that was partitioned into two pools, with the brainstem and rostral spinal cord in the rostral pool that was perfused with a $\mathrm{Ca}^{2+}$-free solution containing AP5 and CNQX to block synaptic transmission (Fig. 3A). Locomotor activity was induced in the caudal part of the spinal cord by NMDA $(100 \mu \mathrm{M})$ and monitored by recording two opposing ventral roots, while descending reticulospinal axons were stimulated using a suction electrode placed at the junction between the brainstem and spinal cord (Fig. 3A). Tetanic stimulation $(5 \times 1 \mathrm{~s}$ at $40 \mathrm{~Hz}$ applied every $2 \mathrm{~s})$ of reticulospinal axons induced a long-term increase in the locomotor frequency of $174.7 \pm 21.7 \%$ of control $(n=6 ; p<0.01)$ (Fig. $3 B, C)$.

To examine whether the long-term potentiation of the locomotor frequency induced by stimulation of descending glutamatergic neurons involves activation of mGluR1, the antagonist CPCCOEt $(100 \mu \mathrm{M})$ was added to the perfusing solution in the 


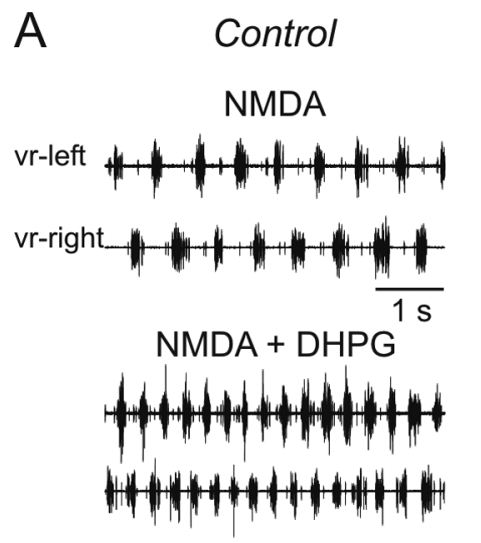

B

AM-251

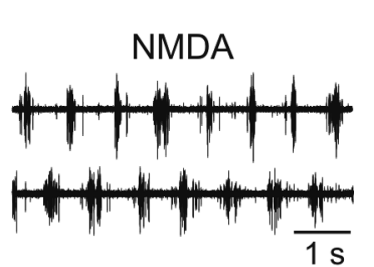

NMDA + DHPG
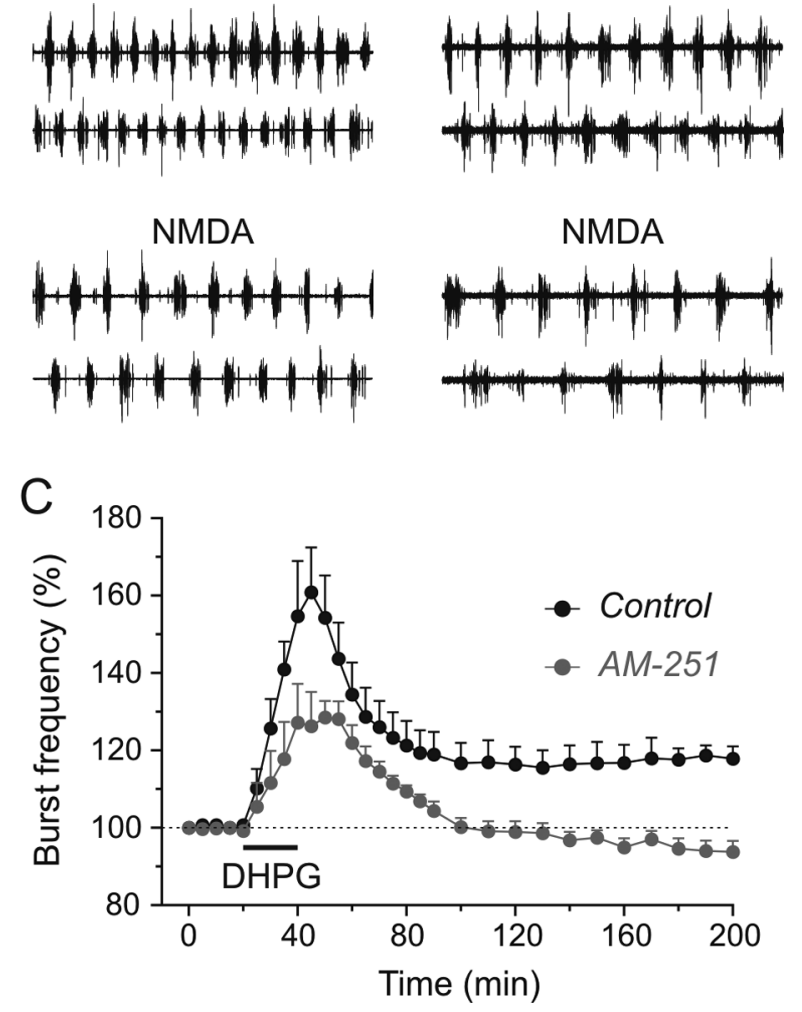

Figure 2. Endocannabinoids mediated the long-term potentiation of the locomotor circuitry. A, Locomotor activity was induced in control preparations by NMDA (100 $\mu \mathrm{M})$ and was recorded from opposing ventral roots (vr-left and vr-right). Application of DHPG (100 $\mu \mathrm{m})$ increased the locomotor frequency that displayed a long-term potentiation lasting after washout. $\boldsymbol{B}$, Blockade of cannabinoid receptors by preincubating the spinal cord with the antagonist AM-251 (1 $\mu \mathrm{M}, 1-2 \mathrm{~h})$ prevented the long-term potentiation induced by DHPG. Only a shortterm increase of the locomotor frequency was induced by DHPG that washed out completely. $C$, Plot of averaged data from different preparations showing the time course of the effect of DHPG on the locomotor frequency. In control preparations, DHPG always induced a short- and longterm potentiation of the locomotor burst frequency. In preparations in which cannabinoid receptors were blocked by $A M-251$, only the short-term increase in the frequency was induced by DHPG and the long-term effect was blocked.

caudal pool. In these preparations, electrical stimulation of reticulospinal axons induced only a transient increase in the locomotor frequency that amounted to $124.9 \pm 18.6 \%(n=5 ; p<$ $0.05)$, whereas the long-term potentiation was completely blocked (95.2 $\pm 1.5 \% ; n=5 ; p>0.05$ ) (Fig. 3C). The contribution of cannabinoid receptor activation to the long-term potentiation induced by stimulation of reticulospinal axons was assessed by using the antagonist AM-251. In the presence of this antagonist tetanic stimulation of descending glutamatergic axons did not induce any long-term increase in the locomotor frequency $(94.6 \pm 1.6 \%$ of control; $n=4 ; p>0.05$ ) (Fig. $3 C$ ). These results show that activation of descending glutamatergic axons induces long-term potentiation of locomotor frequency that is mediated by activation of mGluR 1 and cannabinoid receptors.

Short- and long-term depression of midcycle inhibition

To determine the possible mechanisms responsible for the increase in the locomotor frequency, we examined the effect of mGluR1 activation on midcycle reciprocal inhibition. Locomotor activity was induced by NMDA and monitored by recording two opposing ventral roots while synaptic activity was recorded intracellularly from motoneurons. In control (NMDA alone), the motoneuron received phasic excitation alternating with inhibition and fired action potentials only infrequently (Fig. 4A). Application of DHPG decreased the midcycle reciprocal inhibition resulting in a depolarization of the baseline membrane potential (Fig. $4 B$ ). The depression of the inhibition by DHPG was accompanied by an increase in the locomotor frequency (Fig. $4 D$ ). The synaptic drive received by the motoneuron was averaged and aligned over two locomotor cycles (the action potentials were filtered out), showing a significant decrease in the amplitude of midcycle inhibition by DHPG (Fig. 4C). The decrease in midcycle inhibition in the presence of DHPG was the mirror image of the increase in the locomotor frequency and displayed both a shortand a long-term modulation (Fig. $4 D$ ). The short-term depression of the inhibition was $62.2 \pm 8.1 \%$ of control $(n=6 ; p<$ 0.01 ) whereas the long-term depression was $40.0 \pm 10.3 \%$ of control $(n=6 ; p<0.01)$ and lasted for several hours after washout of DHPG (Fig. 4D). Thus, activation of mGluR1 induces a short- and long-term depression of inhibitory synaptic transmission within the locomotor circuitry with time courses similar to those of the short- and long-term increase in the frequency of the locomotor rhythm.

Furthermore, we tested the effect of DHPG on inhibitory synaptic transmission while ionotropic glutamate receptors were blocked. In these experiments the recording chamber was divided into two pools and fictive locomotion was induced in the rostral part of the spinal cord and monitored by recorded from left and right ventral roots (Fig. $5 \mathrm{~A}$ ). The caudal part of the spinal cord was perfused with high $\mathrm{Ca}^{2+} /$ high $\mathrm{Mg}^{2+}$ solution containing kynurenic acid (2 mM) to block ionotropic glutamate receptors. A motoneuron recorded in the caudal pool showed midcycle inhibitory synaptic input occurring in phase with the contralateral ventral root burst (Fig. 5B). Application of DHPG (100 $\mu \mathrm{M})$ in the caudal pool decreased the amplitude of the inhibitory synaptic potentials by $35.2 \pm 8.4 \%(n=4)($ Fig. $5 B, C)$. These results show that the depression of midcycle inhibition is the results of a direct effect of DHPG on glycinergic synaptic transmission.

Endocannabinoids are required for the short- and long-term depression of mid-cycle inhibition

To test whether endocannabinoids released by activation of mGluR1 are necessary for the short- and long-term depression of midcycle inhibition, the effect of DHPG was tested in preparations preincubated with the cannabinoid receptor antagonist AM-251 $(1 \mu \mathrm{M}, 2 \mathrm{~h})$. Application of DHPG in the presence of AM-251 did not decrease the amplitude of midcycle reciprocal inhibition as seen by the lack of an effect on the baseline membrane potential and the firing of the recorded motoneuron (Fig. $6 A, B)$. The averaged synaptic inputs in the motoneuron showed that there was no significant decrease in the amplitude of the inhibition (Fig. 6C). Blockade of cannabinoid receptors by AM251 counteracted the short-term depression of midcycle inhibition by DHPG and greatly attenuated the long-term depression 
(Fig. 6D). The amplitude of the midcycle inhibition was decreased by $9.1 \pm 12.5 \%$ of control $(n=7 ; p>0.05)$ during the application of DHPG (short-term effect) and by $9.9 \pm 11.7 \%$ of control $(n=7 ; p<$ 0.05 ) after washout of DHPG (long-term effect) (Fig. 6D).

In the preparations pretreated with AM-251, DHPG also failed to induce a long-term potentiation of the locomotor frequency and only a short-term increase was induced (Fig. 6D). The latter amounted to $140.0 \pm 21.6 \%$ of control $(n=7)$ and was significantly less pronounced than in control preparations $(p<0.01$; Fig. $6 D)$. These results show that mGluR1 induces short- and longterm depression of midcycle reciprocal inhibition, both of which are dependent on release of endocannabinoids. The time course of the depression in the inhibitory synaptic transmission is closely related to that of the potentiation of the locomotor frequency, suggesting a link between the modulation of inhibitory synaptic inputs and that of the locomotor circuit activity.

\section{Long-term potentiation of excitatory} synapses during locomotion by mGluR1 To examine whether mGluRl activation also modulates excitatory synaptic transmission to mediate the long-term potentiation of the locomotor frequency, the effect of DHPG was tested on synaptic transmission from excitatory interneurons during fictive locomotion. In these experiments, the recording chamber was divided into two pools by agar and locomotor activity was induced in the rostral pool with NMDA $(100 \mu \mathrm{M})$. Excitatory synaptic transmission from interneurons whose axons project ipsilaterally across the agar barrier was recorded in motoneurons in the caudal pool in the presence of strychnine $(5 \mu \mathrm{M})$ to block glycinergic synaptic transmission but with no NMDA (Fig. 7A). In this configuration the excitatory synaptic transmission is driven by relevant locomotor network activity from the rostral pool. Figure $7 B$ shows a motoneuron recorded in the caudal pool that displayed excitatory synaptic input in phase with the ipsilateral ventral root recorded in the rostral pool. Application of the DHPG $(100 \mu \mathrm{M})$ in the caudal pool increased the amplitude of the excitation without affecting the frequency of the locomotor rhythm recorded in the rostral pool, indicating that the effect of DHPG was restricted to the caudal pool (Fig. 7C,D). The increase in the locomotordriven excitatory synaptic transmission was $151.1 \pm 27.1 \%$ of control $(n=6 ; p<0.01)$ and did not recover after washout of DHPG, thus displaying a long-term potentiation by $131.4 \pm$ $6.4 \%(n=6 ; p<0.01)$ (Fig. $7 E)$.

\section{Endocannabinoids mediate the long-term potentiation of excitatory synaptic transmission}

To test whether the long-term potentiation of excitatory synaptic transmission by DHPG involves release of endocannabinoids, we tested its effect in the presence of AM-251 (1 $\mu \mathrm{M})$. This completely blocked the long-term potentiation $(n=6)$ of the excitatory synaptic transmission (Fig. 8). However, in the presence of
AM-251, DHPG was still able to induce a short-term increase in the excitatory synaptic transmission that recovered to control levels after washout (Fig. $8 A-C$ ). The amplitude of the excitatory synaptic drive increased by $136.5 \pm 38.7 \%$ of control $(n=3)$ by DHPG and recovered completely after washout of the DHPG (Fig. $8 B, C$ ). The short-term effect of DHPG could not be monitored in 3 of the 6 neurons because activation of mGluR 1 in the presence of strychnine induced membrane potential oscillations (cf. Krieger et al., 1998) that precluded the analysis of the amplitude of the excitation during the application of DHPG. These results clearly show that release of endocannabinoids is necessary for the long-term potentiation of excitatory synaptic transmission by mGluR1, whereas the short-term potentiation is independent of endocannabinoids.

\section{The long-term potentiation of excitation is dependent on nitric oxide}

In previous reports, it has been suggested that nitric oxide is involved in endocannabinoid-mediated synaptic plasticity (Makara et al., 2005; Safo and Regehr, 2005). To test whether nitric oxide signaling is involved in mediating the mGluR1induced plasticity of synaptic transmission and locomotor activity, we used a nitric oxide synthase inhibitor. We first examined whether the long-term potentiation of excitatory synaptic transmission by mGluR1 was dependent on nitric oxide. For this a double pool experimental chamber was used (see above) (Fig. $7 A$ ). Preincubation of the caudal pool with the nitric oxide synthesis inhibitor L-NAME $(100 \mu \mathrm{M})$ or the nitric oxide scavenger PTIO $(200 \mu \mathrm{M})$ completely blocked the mGluR1-mediated long- 


\section{Control}

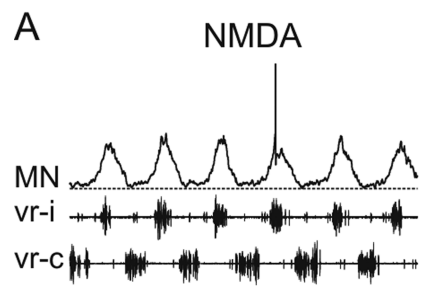

B NMDA + DHPG
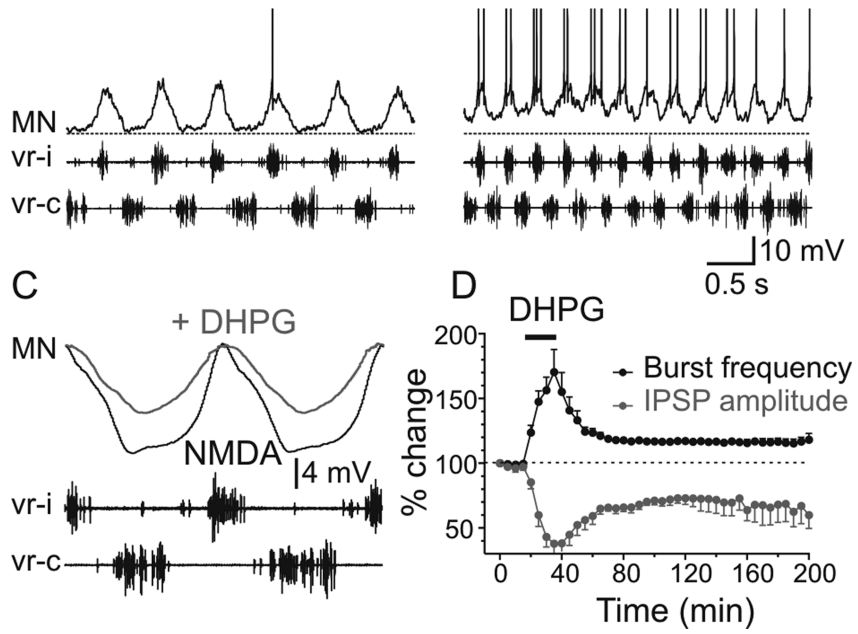

Figure 4. Short- and long-term depression of midcycle inhibition by mGluR1 during locomotion. $A$, Intracellular recording from an $\mathrm{MN}$ that received phasic excitation in phase with the ipsilateral ventral root (vr-i) activity that alternated with phasic inhibition occurring in phase with the contralateral ventral root (vr-c) burst. $\boldsymbol{B}$, Application of DHPG decreased the phasic inhibition and depolarized the baseline membrane potential to increase the firing of the motoneuron. These effects were accompanied by an increase in the locomotor burst frequency. $C$, Averaged synaptic inputs received by the motoneuron aligned with the ipsilateral and contralateral ventral root bursts. The action potentials were filtered out using a low-pass filter. Activation of mGluR1 by DHPG decreased the amplitude of the phasic inhibition whereas the phasic excitation was not decreased. $\boldsymbol{D}$, Averaged data from all the experiments showing the time course of depression of inhibition in parallel with the increase in the locomotor frequency induced by mGluR1. DHPG induced a short- and long-term depression of midcycle inhibition occurring during locomotion that correlated with the short- and long-term potentiation of the frequency.
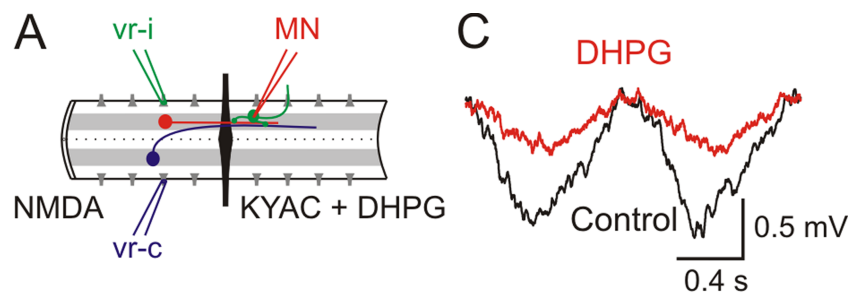

B Control

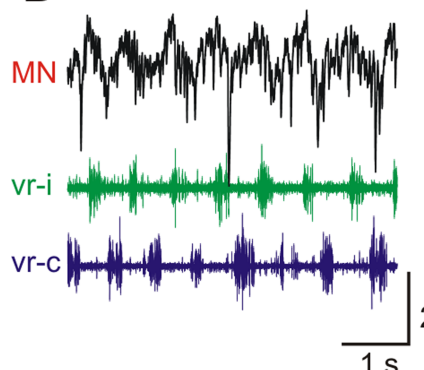

DHPG
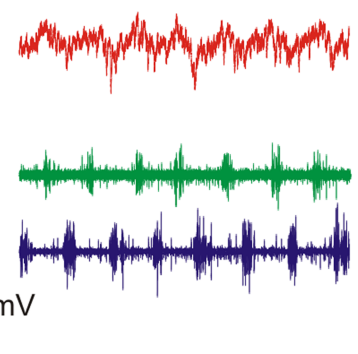

Figure 5. DHPG acts directly to depress inhibitory synaptic transmission. $A$, Experimental setup with double-pool configuration, fictive locomotion was induced in the rostral part of the spinal cord with NMDA and monitored from two opposing ventral roots. Intracellular recording was made from an $\mathrm{MN}$ in the caudal pool that was perfused with a high $\mathrm{Ca}^{2+} / \mathrm{high} \mathrm{Mg}^{2+}$ solution containing KYAC ( $2 \mathrm{mM}$ ) to block ionotropic receptors. $\boldsymbol{B}$, Midcycle inhibition recorded in the $\mathrm{MN}$ occurred in phase with the contralateral ventral root (vr-c) burst and its amplitude was decreased by application of DHPG (100 $\mu \mathrm{M})$ to the caudal pool. C, Averaged inhibitory synaptic input received by the recorded $M N$ in control and in DHPG.
Pre-incubated with AM-251

A NMDA

B NMDA + DHPG
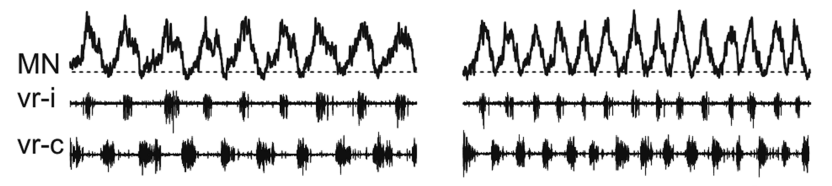

C
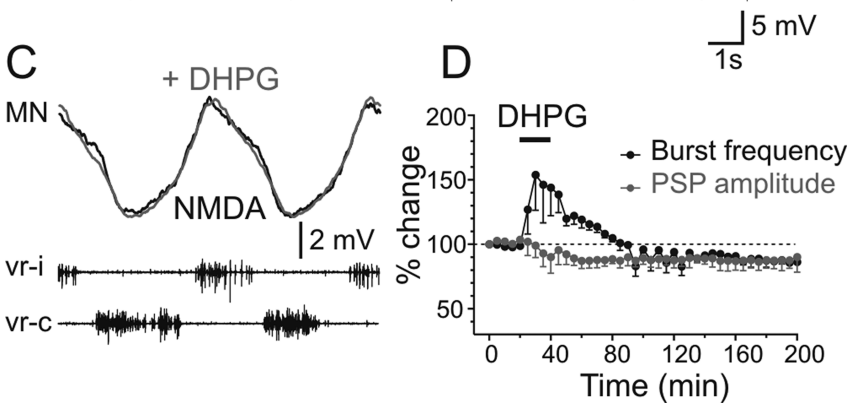

Figure 6. Endocannabinoids mediate the short- and long-term depression of mid-cycle inhibition. $A$, The role of endocannabinoids in mediating the depression of midcycle inhibition by mGluR1 was assessed using the antagonist AM-251. Preparations were preincubated with AM251 and locomotor activity was induced by NMDA while an MN was recorded intracellularly. $\boldsymbol{B}$, Application of DHPG did not significantly change the baseline membrane potential of the MN, but it increased the locomotor frequency. $C$, Averaged synaptic inputs received by $\mathrm{MN}$ in the caudal pool aligned with the ipsilateral (vr-i) and contralateral ventral root (vr-c) activity. DHPG did not decrease the amplitude of the midcycle inhibition. $\boldsymbol{D}$, Averaged data from different preparations showing that blockade of cannabinoid receptors prevented the short- and longterm depression of midcycle inhibition by mGluR1. Only a short-term increase in the locomotor frequency was induced by DHPG.

term potentiation of excitatory synaptic transmission deriving from locomotor network activity in the rostral pool (Fig. 9A,B). In L-NAME, some short-term potentiation of the excitation was still induced by DHPG and amounted to $114.6 \pm 2.6 \%$ of control (Fig. 9A,C). After washout of DHPG the amplitude of the excitatory synaptic transmission recovered to $92.4 \pm 2.1 \%$ of control in L-NAME $(n=4)$ and to $93.1 \pm 1.8 \%$ of control in PTIO $(n=$ 4) (Fig. 9C). Thus, interfering with nitric oxide by using the nitric oxide inhibitor L-NAME or the scavenger PTIO blocked the mGluR1-induced long-term potentiation of excitatory synaptic transmission. These results indicate that in addition to endocannabinoids the mGluR1-induced long-term potentiation of excitatory synaptic transmission also requires nitric oxide synthase activity.

Enhancement of excitatory synaptic transmission by mGluR1 To determine whether the potentiation of excitatory synaptic drive during locomotion is mediated by an increase of transmitter release, we tested the effect of DHPG on monosynaptic EPSPs in the absence of locomotor activity. In these experiments the spinal cord was completely isolated and was perfused with a high $\mathrm{Ca}^{2+} /$ high $\mathrm{Mg}^{2+}$ solution to reduce polysynaptic transmission whereas glycine and NMDA receptors were blocked with strychnine $(5 \mu \mathrm{M})$ and AP5 $(50 \mu \mathrm{M})$, respectively. Intracellular recordings were made from motoneurons and stimulation of the ventral surface of the spinal cord, where axons of excitatory interneurons and reticulospinal neurons project, induced monosynaptic EPSPs that followed high frequency stimulation with a brief, constant delay. Application of DHPG significantly increased the amplitude of the EPSP from $3.2 \pm 0.2 \mathrm{mV}$ in control to $4.2 \pm 0.3 \mathrm{mV}$ $(n=9 ; p<0.001)$ (Fig. 10A). This potentiation of the amplitude of the EPSP persisted for $>1 \mathrm{~h}$ after washout of DHPG (Fig. $10 \mathrm{~B}$ ). 
This increase in the EPSP amplitude was associated with a decrease in the pairedpulse ratio (PPR) from $1.30 \pm 0.03$ in control to $1.08 \pm 0.02$ in DHPG $(n=5 ; p<$ 0.01 ) (Fig. 10C,D). These data suggest that the increase in the EPSP amplitude is mediated by presynaptic mechanisms that enhance glutamate release from presynaptic axons.

To test whether the potentiation of glutamatergic transmission mediated by mGluR1 involves release of endocannabinoids and nitric oxide, the effect of DHPG on monosynaptic EPSPs was examined in the presence of the cannabinoid receptor antagonist AM-251 $(2 \mu \mathrm{M})$ and the nitric oxide synthase inhibitor L-NAME (100 $\mu \mathrm{M})$. In control conditions, DHPG increased the amplitude of the EPSP by $130.2 \pm 2.8 \%$ of control $(n=9)$. In AM251 and L-NAME the DHPG-induced increase in the EPSP was significantly attenuated to $102.3 \pm 0.9 \%(n=6 ; p<0.001)$ and to $108.2 \pm 2.2 \%$ of control $(n=5 ; p<$ 0.01 ), respectively (Fig. $10 \mathrm{E}$ ). These results suggest that the potentiation of excitatory synaptic transmission is mediated by presynaptic mechanisms and involves both endocannabinoids and nitric oxide.

The depression of midcycle inhibition is dependent on nitric oxide

The effects of L-NAME and PTIO were also examined on the mGluR1-induced short- and long-term depression of midcycle reciprocal inhibition. In these experiments the locomotor rhythm was induced by NMDA and monitored by recording activity from two opposing ventral roots while an intracellular recording was made from a motoneuron (Fig. 4). The preparation was preincubated with L-NAME (Fig. $11 A)(100 \mu \mathrm{M})$ or with PTIO (Fig. $11 B$ ) $(200 \mu \mathrm{M})$ and the effect of DHPG was tested on the amplitude of the midcycle inhibition and the locomotor frequency. In L-NAME and PTIO, DHPG always induced a short-term depression of midcycle inhibition by $31.1 \pm 6.0 \%$ of control $(n=$ $9 ; p<0.01)$ and $33.8 \pm 14.1 \%$ of control $(n=5 ; p<0.01)$, respectively. This was paralleled by a short-term potentiation of the locomotor frequency by $135.1 \pm$ $10.1 \%$ of control $(n=9 ; p<0.01)$ in L-NAME and $152.2 \pm 20.6 \%(n=5 ; p<0.01)$ in PTIO (Fig. $11 C, D)$. However, both L-NAME and PTIO blocked the DHPGinduced long-term depression of the inhibition whose amplitude recovered to $94.1 \pm 8.8 \%$ of control $(n=9)$ and to $104.1 \pm 5.5 \%$ of control within 40-60 min of washout, respectively (Fig. $11 C, D)$. In these preparations, DHPG did not induce a long-term potentiation of the locomotor frequency and only a short-term increase in the frequency was obtained that recovered after washout (Fig. 11C,D). These results show that nitric oxide signaling is
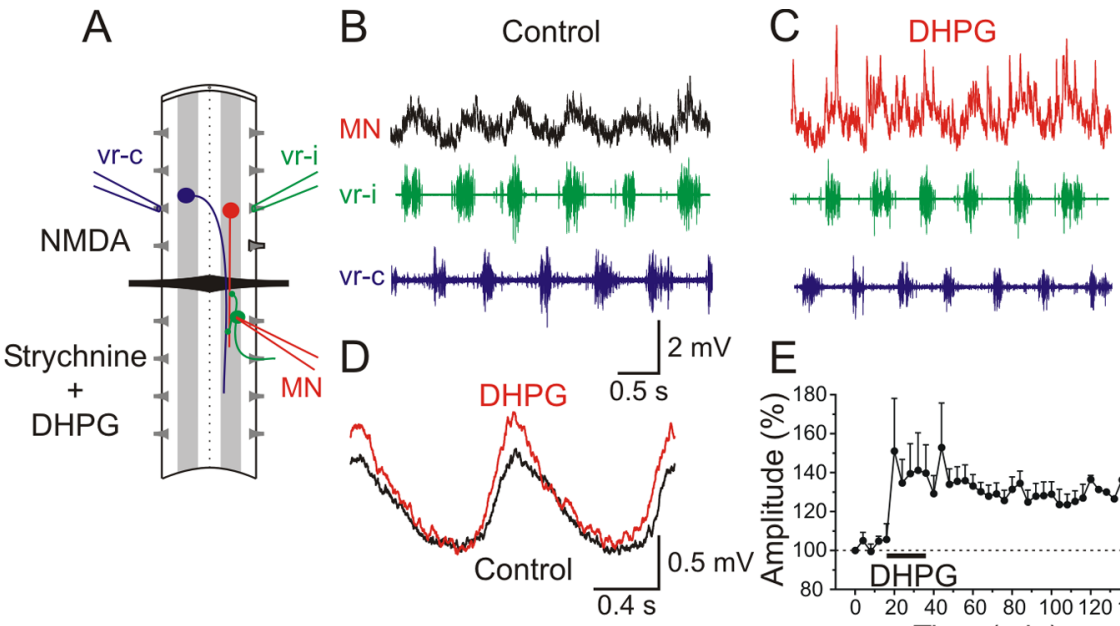

Figure 7. Long-term potentiation of excitatory synaptic transmission during locomotion. $A$, Experimental arrangement with the recording chamber divided into two pools with an agar barrier. In the rostral part of the spinal cord locomotor activity was induced by NMDA while the caudal part was perfused with normal solution containing strychnine to block inhibition and intracellular recording was made from an MN. The red neuron represents ipsilateral excitatory interneurons and the blue neuron represents a commissural inhibitory interneuron. $\boldsymbol{B}$, In controls, the MN received excitation in phase with the ipsilateral ventral root (vr-i) burst. C, Activation of mGluR1 by DHPG in the caudal pool increased the amplitude of the excitatory synaptic input received by the MN. D, Averaged excitatory synaptic input received by the MN showing an increase in amplitude induced by DHPG. $\boldsymbol{E}$, Plot of averaged data from a different experiment showing the time course of the change in the amplitude of the excitation. Application of the DHPG induced a long-term potentiation of excitatory synaptic transmission during locomotion.

\section{Pre-incubated with AM-251}
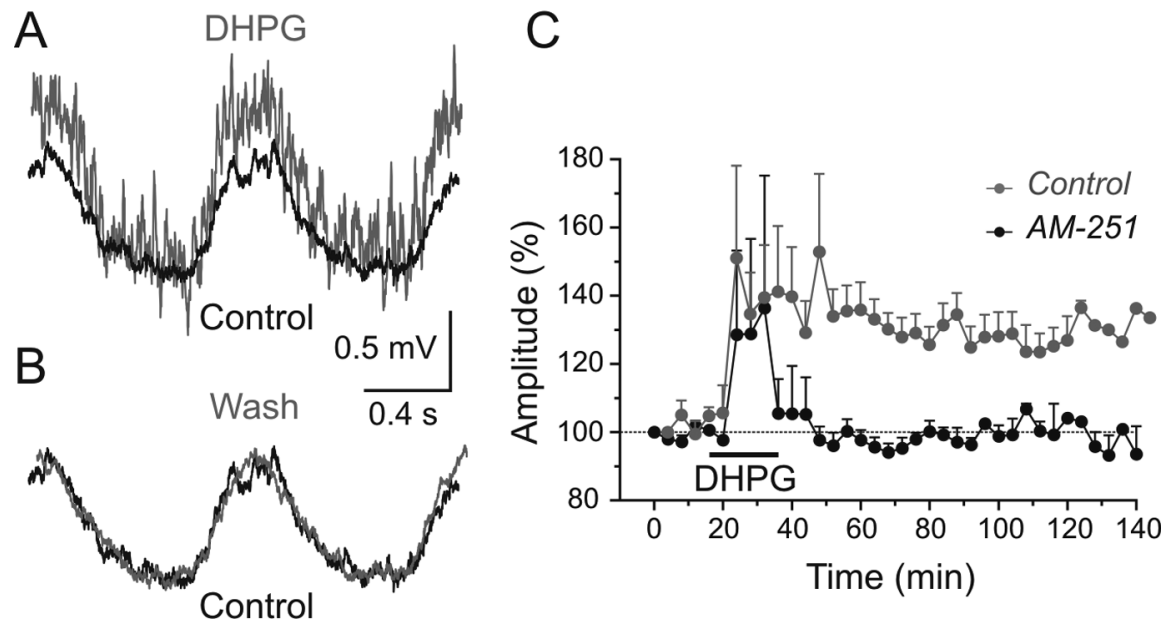

Figure 8. The long-term potentiation of excitation by mGluR1 requires release of endocannabinoids. $\boldsymbol{A}$, Excitatory synaptic transmission was recorded in an MN using the same experimental setup as in Figure 5. Cannabinoid receptors were blocked in the caudal pool by AM-251. Application of DHPG induced only a short-term increase in the amplitude of excitation. $\boldsymbol{B}$, The amplitude of excitatory synaptic transmission recovered to control levels after washout of DHPG. C, Plot of averaged data from preparations in which cannabinoid receptors were blocked in the caudal pool. Application of DHPG induced only a short-term, but no long-term potentiation of the amplitude of the excitation. For comparison the graph from control preparations is also plotted (gray dots). necessary for the mGluR1-mediated long-term, but not the short-term depression of the midcycle inhibition during locomotion.

\section{Nitric oxide is required for long-term potentiation of} locomotor frequency

In these experiments the effect of DHPG on the locomotor frequency was first tested in the presence of L-NAME and subsequently after washout of L-NAME in the same preparations. In 


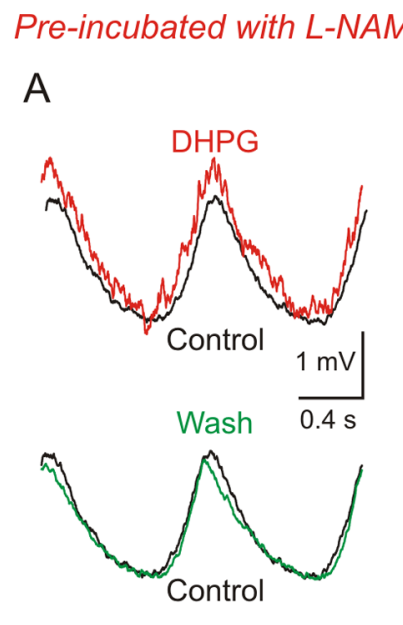

Pre-incubated with PTIO

B

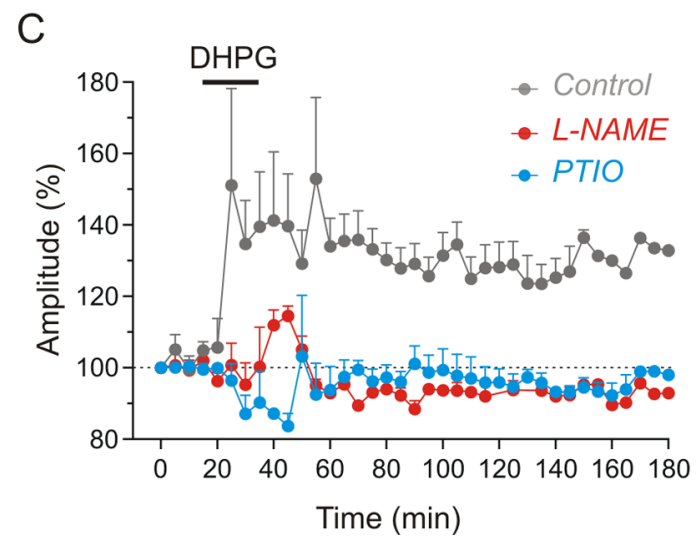

Figure 9. mGluR1-mediated long-term potentiation of excitation requires nitric oxide signaling. Using a double-pool experimental setup (Fig. 6), we tested the role of nitric oxide in mediating the long-term potentiation of excitation by DHPG. In these experiments, the caudal pool was preincubated with the nitric oxide synthase inhibitor L-NAME or the scavenger PTIO.A, L-NAME blocked the long-term potentiation of excitation induced by DHPG. B, PTIO also blocked the long-term potentiation of excitation by mGluR1. C, Plot of averaged data from different experiments in which the caudal pool L-NAME (red graph) or PTIO (blue graph) before application of DHPG. Gray graph shows the effect of DHPG on the amplitude of excitation in control.

the presence of L-NAME, DHPG induced no long-term, but a short-term increase in the locomotor frequency that amounted to $138.1 \pm 9.7 \%$ of control $(n=7 ; p<0.01)$ (Fig. $12 A, C)$. The frequency always recovered within $40 \mathrm{~min}$ after washout of DHPG (Fig. 12A,C). When L-NAME had been washed out, a second application of DHPG induced a larger short-term increase in the frequency by $165.1 \pm 10.7 \%$ of control $(n=7 ; p<$ 0.01 ) (Fig. $12 B, C$ ) that was always followed by a long-term potentiation by $122.1 \pm 1.2 \%$ of control $(n=7 ; p<0.01)$ (Fig. $12 \mathrm{C})$. The long-term potentiation persisted for more that $2-3 \mathrm{~h}$ after washout of DHPG (Fig. 12C).

An established signal transduction pathway for nitric oxide is stimulation of soluble guanylyl cyclase leading to cGMP accumulation. To test for the involvement of this pathway in the potentiation of the locomotor frequency by mGluR1, we used the soluble guanylyl cyclase inhibitor ODQ. In these experiments, the spinal cord was first preincubated in ODQ ( $5 \mu \mathrm{M}, 2 \mathrm{~h})$ before testing the DHPG effect. In the presence of ODQ, DHPG induced only a short-term increase in the locomotor frequency of $144.6 \pm$ $3.1 \%(n=4 ; p<0.01)$, but no long-term potentiation of the frequency (Fig. 12C). These results indicate that nitric oxide is necessary for the mGluR1-induced long-term potentiation of the locomotor frequency, although it only contributes to the shortterm increase.
A

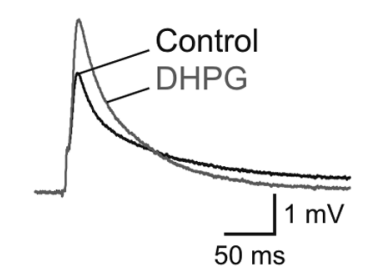

$\mathrm{B}$
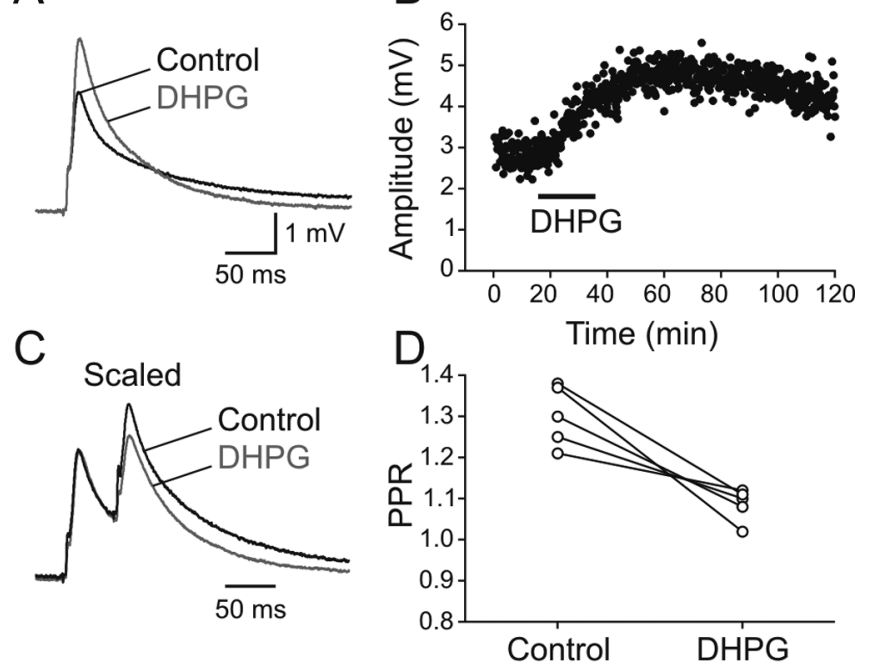

E

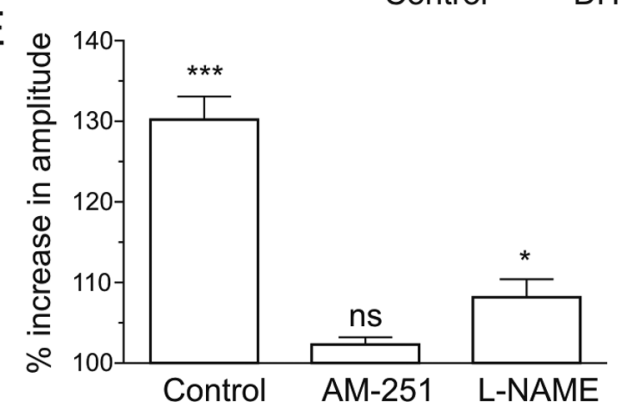

Figure 10. Excitatory synaptic transmission is potentiated via presynaptic mechanisms. $\boldsymbol{A}$, The amplitude of the EPSPs elicited in a MN by stimulation of excitatory axons was enhanced by DHPG. The traces are averages of 30 sweeps. $\boldsymbol{B}$, Plot of the EPSP amplitude before and after DHPG application. $C$, The increase in the amplitude of the EPSP was accompanied by a decrease in the paired-pulse ratio. The trace in DHPG was scaled to the first EPSP in control. Traces are averages of 30 sweeps. D, The paired-pulse ratio was significantly decreased in DHPG compared with control. $E$, Plot showing the percent increase in the EPSP amplitude in control that was attenuated by the cannabinoid receptor antagonist AM-251 and the nitric oxide synthase inhibitor L-NAME. ${ }^{*} p<0.05 ;{ }^{* * *} p<0.001$.

\section{Discussion}

Plasticity of the spinal locomotor circuitry

In this study, we were able to examine the mechanisms of longterm synaptic plasticity during ongoing circuit activity and its consequence on the integrated motor pattern underlying locomotion. The spinal locomotor circuitry allows the modulation of synaptic transmission to be linked to network plasticity. In the lamprey the organization of the circuitry has been characterized in some detail (Buchanan, 2001; Grillner, 2003). As in other vertebrates, the locomotor pattern consists of ipsilateral excitation alternating with midcycle reciprocal inhibition. Locomotor activity, corresponding to swimming in the intact animal, can be induced in the isolated spinal cord in vitro (Wallen and Williams, 1984). Thus, the firing frequency and activation phase of circuit interneurons is not imposed by external stimulation but results from integrated circuit activity. The strength of both inhibitory and excitatory synaptic transmission can be monitored separately and correlated with the overall change of the locomotor circuit activity.

Our results show that mGluR1 activation induces both shortand long-term plasticity by changing the balance between excitation and inhibition within the locomotor circuitry. A brief activation of mGluR1 induces a long-term potentiation of the locomotor frequency that can last $>24 \mathrm{~h}$. This potentiation is 


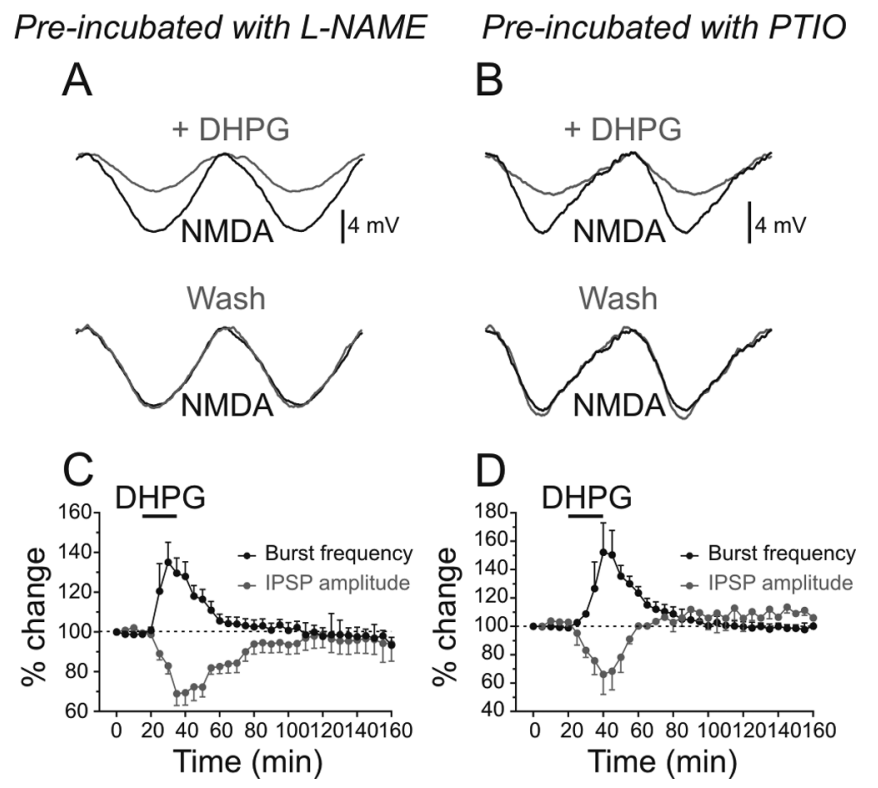

Figure 11. Nitric oxide is required for long-term, but not the short-term depression of midcycle inhibition during locomotion. $A$, In a preparation preincubated with L-NAME, activation of mGluR1 by DHPG decreased the amplitude of midcycle inhibition, which recovered to control level after washout. $\boldsymbol{B}$, In the presence of PTIO, DHPG also transiently decreased the amplitude of midcycle inhibition, which recovered after washout. $\boldsymbol{C}$, Plot of averaged data from different experiments in which the effect of DHPG was tested on midcycle inhibition and locomotor frequency in the presence of L-NAME. DHPG induced only a short-term, but no long-term depression of midcycle inhibition. Similarly, the locomotor frequency displayed only a short-term, but no long-term potentiation. D, Plot showing the block by PTIO of the long-term effects of DHPG on the midcycle inhibition and locomotor frequency.

mediated by a concomitant long-term depression of midcycle reciprocal inhibition and long-term potentiation of ipsilateral excitation arising from locomotor circuit neurons. Blockade of cannabinoid receptors and nitric oxide signaling prevented the mGluR1-mediated long-term plasticity of inhibitory and excitatory synaptic transmission as well as that of the locomotor circuit activity. These results show that the spinal cord is endowed with memory mechanisms requiring release of both endocannabinoids and nitric oxide that transform a brief activation of mGluR1 into long-term potentiation of the locomotor networks.

Correlation between mGluR1-mediated long-term depression of inhibition and long-term potentiation of locomotor frequency and their dependence on endocannabinoids

In the lamprey spinal cord, mGluR1s are localized postsynaptically (Krieger et al., 1998; El Manira et al., 2002). We showed previously that activation of mGluR1 induces a short-term increase in the locomotor frequency by a combination of three mechanisms: (1) inhibition of a leak current, (2) potentiation of NMDA receptors, and (3) release of endocannabinoids (Krieger et al., 2000; El Manira et al., 2002; Kettunen et al., 2005). In the quiescent spinal cord, using artificial stimulation of commissural inhibitory interneurons, we showed that activation of mGluR1 depresses inhibitory synaptic transmission via release of endocannabinoids (Kettunen et al., 2005). However, because in these previous experiments the locomotor network was not activated it was not possible to determine whether the midcycle inhibition was depressed and how its time course correlates with the increase in the locomotor frequency.

Our present results show that activation of mGluR1 induces not only a short- but also a long-term depression of the midcycle

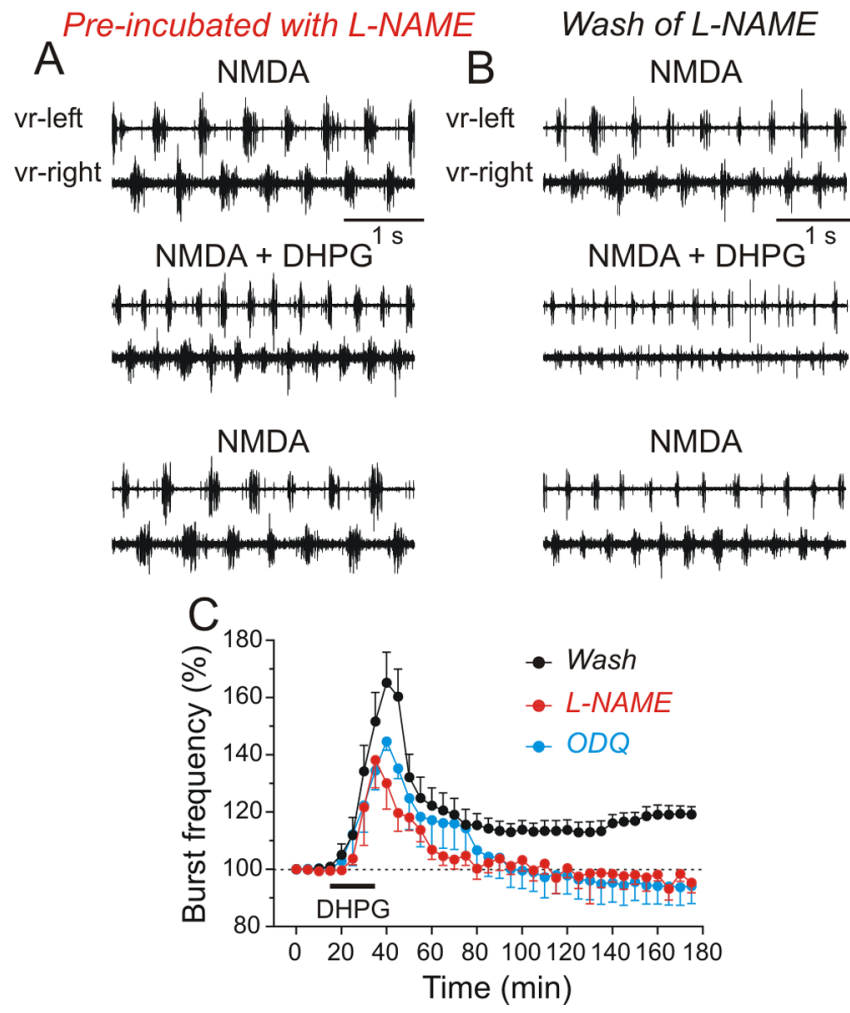

Figure 12. Nitric oxide is involved in mediating the long-term potentiation of the locomotor frequency by mGluR1. $A$, In a preparation preincubated with L-NAME, application of DHPG only transiently increased the locomotor frequency which recovered to control levels after washout. $\boldsymbol{B}$, A second application of DHPG in the same preparation after washout of L-NAME induced an increase in the locomotor frequency which did not recover to control level after washout. $\boldsymbol{C}$, Averaged data from different experiments showing the time course of the change in the locomotor frequency before, during and after application of DHPG. In the presence of L-NAME, DHPG induced only a short-, but no long-term potentiation of the frequency (red graph). After washout of L-NAME, a second application of DHPG induced both short-and long-term potentiation of the locomotor frequency. Similarly, the soluble guanylyl cyclase inhibitor ODQ also blocked the long-term potentiation of the locomotor frequency (blue graph).

inhibition resulting from the appropriate activation of commissural interneurons. The depression of the inhibition represents a mirror image of the short- and long-term potentiation of the locomotor frequency. Blockade of cannabinoid receptors blocked both the short- and long-term depression of the midcycle inhibition although it only abolished the long-term potentiation of the locomotor frequency induced by activation of mGluR1. Thus, the time course of the depression of the inhibitory synaptic transmission matches precisely that of the potentiation of the locomotor frequency suggesting that the modulation of the inhibition is involved in mediating the long-term change in the activity of the locomotor network.

Endocannabinoids have been shown to mediate short-term plasticity of synaptic transmission in many regions of the CNS including the spinal cord (Wilson and Nicoll, 2002; Freund et al., 2003; Kettunen et al., 2005; Chevaleyre et al., 2006; Safo et al., 2006). In most cases, a large depolarization of postsynaptic neurons was necessary to release endocannabinoids that acted as retrograde messengers to depress inhibition or excitation by inhibiting calcium influx in presynaptic terminals. Endocannabinoids could also be released by activation of G-protein receptors such as group $1 \mathrm{mGluRs}$ or muscarinic receptors and depress synaptic transmission (Maejima et al., 2001; Kim et al., 2002; Kettunen et al., 2005). Previously, endocannabinoid-mediated 
long-term synaptic plasticity has been reported in many brain structures and exclusively consists of depression of synaptic transmission (see Chevaleyre et al., 2006). Although in several cases the cellular mechanisms responsible for endocannabinoiddependent short- and long-term synaptic plasticity have been partially elucidated, their functional relevance to the operation of neural circuitry or behavior is still mostly unknown. This is because circuitry in slice preparations is usually quiescent and the synaptic inputs studied are often induced by artificial stimulation that does not necessarily reflect the normal pattern of activity of the circuitry in vivo.

\section{Requirement of endocannabinoids for mGluR1-mediated potentiation of excitatory synaptic transmission during locomotion}

One major novel finding of this study is that endocannabinoids released by activation of $\mathrm{mGluR} 1$ induce long-term potentiation of excitatory synaptic transmission arising from activity of network interneurons during locomotion. Thus far, all known forms of synaptic plasticity mediated by endocannabinoids correspond to depression of synaptic transmission (Chevaleyre et al., 2006). Our results represent the first demonstration of a long-term potentiation of excitatory synaptic transmission via presynaptic mechanisms that requires endocannabinoid release. The potentiation of the excitatory synaptic transmission by endocannabinoids released by mGluR1 may contribute to the long-term potentiation of the locomotor frequency. The endocannabinoidindependent short-term potentiation of excitatory synaptic transmission may involve direct effect of mGluR1 on leak current and NMDA receptors (Cochilla and Alford, 1998; Krieger et al., 2000; Kettunen et al., 2003). Finally, activation of mGluR1 enhances excitation even when glycinergic inhibition is blocked with strychnine suggesting that the potentiation is not attributable to an increase in the excitatory drive caused by disinhibition of excitatory interneurons.

\section{Nitric oxide is necessary for long-term plasticity}

The long-term plasticity of the locomotor frequency as well as of the inhibitory and excitatory synaptic transmission by mGluR 1 is also dependent on nitric oxide. The effect of nitric oxide synthase inhibition on the mGluR1-mediated modulation of the locomotor frequency and excitatory synaptic transmission is similar to that of the cannabinoid receptor blocker, AM-251. However, nitric oxide synthase and cannabinoid receptor blockers differ in their action on the mGluR1-induced depression of the inhibitory synaptic transmission. Whereas blockade of cannabinoid receptors blocked both short- and long-term depression of the inhibition by mGluR1, inhibition of the synthesis of nitric oxide only counteracted the long-term depression leaving the short-term decrease of the inhibition unblocked. This indicates that mGluR1 activates separate mechanisms to induce the short- and longterm plasticity of mid-cycle inhibition with nitric oxide signaling being required for only the long-term depression.

Nitric oxide signaling, in addition to endocannabinoids, is necessary for the long-term plasticity mediated by mGluR1 both on synaptic transmission and on the overall locomotor circuit activity. Endocannabinoids and nitric oxide seem to be acting in concert to mediate the long-term plasticity. In Xenopus tadpoles, nitric oxide has been shown to inhibit swimming activity by enhancing inhibitory synaptic transmission, without an obvious effect on excitation (McLean and Sillar, 2002). These effects of nitric oxide on glycinergic inhibition in the spinal cord are mediated indirectly by modulating endogenous spinal noradrena- line release (McLean and Sillar, 2004). Although our results show that endocannabinoids and nitric oxide act synergistically to produce the long-term plasticity, it is not yet clear how these messengers act in relation to each other. We showed previously that endocannabinoids are released from motoneurons and interneurons and act presynaptically to depress inhibitory synaptic transmission (Kettunen et al., 2005). It has been suggested that, in hippocampus and cerebellum, both nitric oxide and endocannabinoid signaling are involved in mediating synaptic plasticity. (Makara et al., 2005; Safo and Regehr, 2005). It has been suggested that in cerebellum nitric oxide may be acting downstream from endocannabinoids and may be released from presynaptic terminals and act as an anterograde messenger (Safo and Regehr, 2005). However, nitric oxide in hippocampus has been suggested to act upstream from endocannabinoids (Makara et al., 2005). In the spinal locomotor circuitry, it is not yet known if nitric oxide is released from presynaptic terminals or postsynaptic neurons. However, what is clear from our data are that both endocannabinoids and nitric oxide constitute two necessary regulatory steps required for inducing long-term synaptic and circuit plasticity mediated by mGluR1. Future studies are necessary to clarify the interactions between endocannabinoids and nitric oxide and their role in regulating the activity within the locomotor circuitry.

\section{Long-term modulation of the spinal locomotor circuitry and recovery of function after injury}

Although the spinal cord contains the necessary circuitry that is able to generate coordinated locomotor movements, this ability is severely disrupted after spinal cord injury. This is mainly attributable to the lack of excitatory inputs that are normally provided by descending neurons from locomotor centers in the forebrain and brainstem (Edgerton et al., 2004; Frigon and Rossignol, 2006). Our results show the existence of long-term plasticity intrinsic to the locomotor circuitry, whereby a brief activation of mGluR1 is "memorized" for a long period. This "memory" mechanism is dependent on endocannabinoids and nitric oxide signaling and is the result of a shift in balance between inhibition and excitation during the activity of the locomotor networks. Because both descending supraspinal neurons and sensory neurons use glutamate as transmitter, it is most likely that the released glutamate during locomotion in the intact system will not only activate ionotropic receptors but also metabotropic receptors to modulate in an activity-dependent manner synaptic transmission and the overall activity of the locomotor circuitry. It is now clear that motor training can promote recovery of function after spinal cord injury in both animals and humans by making use of the sensory feedback activated by the stepping (Dietz and Harkema, 2004; Edgerton et al., 2004; Frigon and Rossignol, 2006). This recovery is gradual and may result in a dynamic reconfiguration of the locomotor circuitry that leads to a long-term shift in the balance between inhibitory and excitatory synaptic transmission from network interneurons. mGluR1-mediated recruitment of endocannabinoids and nitric oxide signaling could play an important role in this reconfiguration.

\section{References}

Abbott LF, Regehr WG (2004) Synaptic computation. Nature 431:796-803. Buchanan JT (1982) Identification of interneurons with contralateral, caudal axons in the lamprey spinal cord: synaptic interactions and morphology. J Neurophysiol 47:961-975.

Buchanan JT (2001) Contributions of identifiable neurons and neuron classes to lamprey vertebrate neurobiology. Prog Neurobiol 63:441-466. Buchanan JT, Grillner S (1987) Newly identified "glutamate interneurons” 
and their role in locomotion in the lamprey spinal cord. Science 236:312-314.

Chevaleyre V, Takahashi KA, Castillo PE (2006) Endocannabinoidmediated synaptic plasticity in the CNS. Annu Rev Neurosci 29:37-76.

Cochilla AJ, Alford S (1998) Metabotropic glutamate receptor-mediated control of neurotransmitter release. Neuron 20:1007-1016.

Dale N (1985) Reciprocal inhibitory interneurones in the Xenopus embryo spinal cord. J Physiol (Lond) 363:61-70.

Dale N, Grillner S (1986) Dual-component synaptic potentials in the lamprey mediated by excitatory amino acid receptors. J Neurosci 6:2653-2661.

Dale N, Roberts A (1985) Dual-component amino-acid-mediated synaptic potentials: excitatory drive for swimming in Xenopus embryos. J Physiol (Lond) 363:35-59.

Dan Y, Poo MM (2006) Spike timing-dependent plasticity: from synapse to perception. Physiol Rev 86:1033-1048.

Destexhe A, Marder E (2004) Plasticity in single neuron and circuit computations. Nature 431:789-795.

Dietz V, Harkema SJ (2004) Locomotor activity in spinal cord-injured persons. J Appl Physiol 96:1954-1960.

Edgerton VR, Tillakaratne NJ, Bigbee AJ, de Leon RD, Roy RR (2004) Plasticity of the spinal neural circuitry after injury. Annu Rev Neurosci 27:145-167.

El Manira A, Kettunen P, Hess D, Krieger P (2002) Metabotropic glutamate receptors provide intrinsic modulation of the lamprey locomotor network. Brain Res Brain Res Rev 40:9-18.

Feldman JL, Mitchell GS, Nattie EE (2003) Breathing: rhythmicity, plasticity, chemosensitivity. Annu Rev Neurosci 26:239-266.

Freund TF, Katona I, Piomelli D (2003) Role of endogenous cannabinoids in synaptic signaling. Physiol Rev 83:1017-1066.

Frigon A, Rossignol S (2006) Functional plasticity following spinal cord lesions. Prog Brain Res 157:231-260.

Garthwaite J, Boulton CL (1995) Nitric oxide signaling in the central nervous system. Annu Rev Physiol 57:683-706.

Grillner S (2003) The motor infrastructure: from ion channels to neuronal networks. Nat Rev Neurosci 4:573-586.

Grillner S, McClellan A, Sigvardt K, Wallen P, Wilen M (1981) Activation of NMDA-receptors elicits "fictive locomotion" in lamprey spinal cord in vitro. Acta Physiol Scand 113:549-551.

Kettunen P, Krieger P, Hess D, El Manira A (2002) Signaling mechanisms of metabotropic glutamate receptor 5 subtype and its endogenous role in a locomotor network. J Neurosci 22:1868-1873.

Kettunen P, Hess D, El Manira A (2003) mGluR1, but not mGluR5, mediates depolarization of spinal cord neurons by blocking a leak current. J Neurophysiol 90:2341-2348.

Kettunen P, Kyriakatos A, Hallen K, El Manira A (2005) Neuromodulation via conditional release of endocannabinoids in the spinal locomotor network. Neuron 45:95-104.
Kiehn O (2006) Locomotor circuits in the mammalian spinal cord. Annu Rev Neurosci 29:279-306.

Kim J, Isokawa M, Ledent C, Alger BE (2002) Activation of muscarinic acetylcholine receptors enhances the release of endogenous cannabinoids in the hippocampus. J Neurosci 22:10182-10191.

Kreitzer AC, Regehr WG (2001) Retrograde inhibition of presynaptic calcium influx by endogenous cannabinoids at excitatory synapses onto Purkinje cells. Neuron 29:717-727.

Krieger P, Grillner S, El Manira A (1998) Endogenous activation of metabotropic glutamate receptors contributes to burst frequency regulation in the lamprey locomotor network. Eur J Neurosci 10:3333-3342.

Krieger P, Hellgren-Kotaleski J, Kettunen P, El Manira AJ (2000) Interaction between metabotropic and ionotropic glutamate receptors regulates neuronal network activity. J Neurosci 20:5382-5391.

Maejima T, Hashimoto K, Yoshida T, Aiba A, Kano M (2001) Presynaptic inhibition caused by retrograde signal from metabotropic glutamate to cannabinoid receptors. Neuron 31:463-475.

Makara JK, Katona I, de Vente, Freund TF, Hajos N (2005) Interaction of nitric oxide and cannabinoid signaling in hippocampal depolarizationinduced suppression of inhibition. Soc Neurosci Abstr 31:720.8.

Malenka RC, Bear MF (2004) LTP and LTD: an embarrassment of riches. Neuron 44:5-21.

Marder E, Goaillard JM (2006) Variability, compensation and homeostasis in neuron and network function. Nat Rev Neurosci 7:563-574.

McLean DL, Sillar KT (2002) Nitric oxide selectively tunes inhibitory synapses to modulate vertebrate locomotion. J Neurosci 22:4175-4184.

McLean DL, Sillar KT (2004) Metamodulation of a spinal locomotor network by nitric oxide. J Neurosci 24:9561-9571.

McLean DL, Merrywest SD, Sillar KT (2000) The development of neuromodulatory systems and the maturation of motor patterns in amphibian tadpoles. Brain Res Bull 53:595-603.

Micheva KD, Buchanan J, Holz RW, Smith SJ (2003) Retrograde regulation of synaptic vesicle endocytosis and recycling. Nat Neurosci 6:925-932.

Prast H, Philippu A (2001) Nitric oxide as modulator of neuronal function. Prog Neurobiol 64:51-68.

Ramirez JM, Viemari JC (2005) Determinants of inspiratory activity. Respir Physiol Neurobiol 147:145-157.

Safo PK, Regehr WG (2005) Endocannabinoids control the induction of cerebellar LTD. Neuron 48:647-659.

Safo PK, Cravatt BF, Regehr WG (2006) Retrograde endocannabinoid signaling in the cerebellar cortex. Cerebellum 5:134-145.

Wallen P, Williams TL (1984) Fictive locomotion in the lamprey spinal cord in vitro compared with swimming in the intact and spinal animal. J Physiol (Lond) 347:225-239.

Wilson RI, Nicoll RA (2002) Endocannabinoid signaling in the brain. Science 296:678-682.

Wilson RI, Kunos G, Nicoll RA (2001) Presynaptic specificity of endocannabinoid signaling in the hippocampus. Neuron 31:453-462. 